\title{
Prolongation of Insulin Removal by Perfused Liver from Spontaneously Obese Rats
}

\author{
D. Weiland, C. E. Mondon, and G. M. Reaven \\ Department of Medicine, Stanford University School of Medicine and Veterans Administration Hospital, Palo Alto, California, USA
}

\begin{abstract}
Summary. The $t_{1 / 2}$ of insulin removal by perfused liver from hyperinsulinaemic ob/ob mice has previously been shown to be prolonged as compared to livers from nonobese mice. Since this defect improved when insulin levels were lowered in vivo, it appeared that the abnormality might be secondary to hyperin-
\end{abstract} sulinaemia. To test this hypothesis further, insulin removal by perfused liver from one year and 6 week old Sprague-Dawley rats has been compared. Older rats were both obese and hyperinsulinaemic, and their $t_{1 / 2}$ of insulin removal (mean \pm SEM) was significantly prolonged $(11.0 \pm 0.56 \mathrm{~min}$ vs $7.6 \pm$ $0.29 \mathrm{~min}$ ). In addition, the induction of severe streptozotocin-induced hypoinsulinaemia in young rats led to a shortening of the $t_{1 / 2}(5.56 \pm 0.72 \mathrm{~min})$. However, the $t_{1 / 2}$ also fell comparably when weight gain of young control rats was limited to that of young hypoinsulinaemic rats by caloric restriction (5.89 \pm $0.81 \mathrm{~min}$ ). Furthermore, $\mathrm{t}_{1 / 2}$ was not increased when young rats were made hyperinsulinaemic by administration of NPH insulin for 12-14 days, nor was it decreased when older rats were made moderately hypoinsulinaemic by the administration of streptozotocin. These results demonstrate that the defect in hepatic insulin removal observed in the ob/ ob mouse is not unique to this genetic variant, but is also observed in normal obese rats. Although the mechanism responsible for this phenomenon remains to be defined, it does not seem to be a simple function of relatively short-term (1-2 weeks) changes in plasma insulin concentration. On the other hand, it is still possible that the prolongation of insulin removal in both the ob/ob mouse and the obese rat is secondary to more chronic elevations in plasma insulin, or, alternatively is related to the obese state itself. In either event, the defect in hepatic insulin removal may be a general feature of obesity and, as such, contribute to the maintenance of hyperinsulinaemia in a variety of states characterized by obesity.

Key words: Perfused liver, hepatic insulin removal, obesity, hyperinsulinaemia, hypoinsulinaemia.

It has recently been shown that the half-time $\left(t_{1 / 2}\right)$ of insulin removal by perfused liver from obesehyperglycaemic (ob/ob) mice was prolonged as compared with the $t_{1 / 2}$ found for livers from nonobese mice [1]. As a result, more insulin was found in the perfusate leaving the liver of ob/ob mice than in that of the controls. This anomalous handling of insulin by livers of ob/ob mice appeared to be corrected by inducing hypoinsulinaemia with streptozotocin, antiinsulin serum, or prolonged fasting. On the basis of these findings it was suggested that a primary rise in ambient insulin levels in ob/ob mice might lead to a defect in hepatic insulin removal, which, in turn, would tend to perpetuate the hyperinsulinaemia. The combination of hyperinsulinaemia and obesity is not confined to the ob/ob mouse, and the relevance of the vicious circle postulated above to the obese human is self-evident. Therefore, it seemed important to see if this defect in hepatic insulin metabolism was a genetic lesion, unique to the ob/ob mouse, or was generally associated with obesity and hyperinsulinaemia. In order to do this, we chose to study the one year old rat, in which both obesity [2] and hyperinsulinaemia [3] develop spontaneously. In these experiments, we have used the technique of isolated recycling perfusion to compare the removal of insulin by livers from 6 week old Sprague-Dawley rats to that of livers from one year old rats of the same species. 


\section{Materials and Methods}

\section{Animals}

All liver donors were male Sprague-Dawley rats. Two basic groups of rats were used for all experiments. One group consisted of young rats, 6 weeks old, weighing $150-200 \mathrm{~g}$. The other group of rats were one year old and weighed more than $550 \mathrm{~g}$. Four series of experients were performed. In the first series (A) untreated old and young rats were compared. In Study B, a group of young rats were made chronically hyperinsulinaemic by $8 \mathrm{AM}(1 / 3$ of daily dose) and $8 \mathrm{PM}(2 / 3$ of daily dose) injections of subcutaneous NPH insulin for 12-14 days. Animals were initially injected with a daily dose of $1.5 \mathrm{U}$ of insulin. Insulin dosage was raised $1.0 \mathrm{U}$ per day until the fifth day, after which a plateau level of $5.5 \mathrm{U} /$ day was maintained. Controls received injections of NPH diluent fluid. In another series of experiments (C) a group of old rats were made hypoinsulinaemic and hyperglycaemic by intravenous tail vein injections of streptozotocin (Upjohn Co., lot $\neq 60140$ ) (in $0.02 \mathrm{M}$ citrate buffer solution, $\mathrm{pH} 4.5$ ) at a dosage of $22 \mathrm{mg} / \mathrm{kg}$ rat weight. Controls were injected with the citrate buffer solution. In the final series of experiments (D), two groups of young rats were made diabetic with streptozotocin as described above. Rats received either $50 \mathrm{mg}$ or $80 \mathrm{mg}$ of streptozotocin per $\mathrm{kg}$ body weight, depending upon degree of hyperglycaemia and hypoinsulinaemia desired. A third group of young rats were fed a calorically restricted diet, which allowed neither weight gain nor loss. Control animals were again injected with citrate buffer solution. Plasma glucose and insulin levels were determined after an overnight fast in all rats. For all diabetic animals and respective controls, this was done $72 \mathrm{hr}$ post-injection. For insulin injected animals and controls of Study B, this was done on the 8th day of insulin injections. Young calorically restricted animals underwent an overnight fast on the 6th day of their diet. Fasting blood was obtained by wrapping conscious animals in a terry cloth towel and clipping the exposed tip of the tail. Blood samples were drawn into microcapillary tubes previously rinsed with 5\% EDTA. Following the overnight fast all animals, except calorically restricted ones, were allowed free access to food for three days to recover the weight loss and resume normal daily weight gain prior to perfusion. Young calorically restricted animals were fed ad lib for 2 days after the fast until they had regained their initial weight and then placed on their calorically restricted diet for 5 days prior to perfusion.

\section{Perfusion Technique}

All perfusions were started in the morning, and both liver and blood donors were anaesthetized with sodium thiamylal $(6.2 \mathrm{mg} /$ $100 \mathrm{~g}$ body weight) prior to surgery. The perfusing medium consisted of six parts defibrinated rat blood obtained from large male Sprague-Dawley rats, and four parts KRB (Krebs-Ringer bicarbonate), buffer containing $3 \mathrm{~g} / 100 \mathrm{ml}$ bovine albumin (Reheis Chemical, Chicago). The medium was adjusted to a $\mathrm{pH}$ of 7.35 . The hematocrit of the medium was approximately $27 \%$. Livers were perfused in situ, with recycled medium, using previously described techniques $[4,5]$. At the start of recirculation, flow rates were adjusted to $1.0 \mathrm{ml} / \mathrm{min}$ per g estimated wet liver weight. Weight was estimated from the total body weight of the animal prior to the infusion. Estimates were based on our own unpublished series of liver to body weights ( $>200$ young rats, and $>50$ old rats), where we found liver weight to be $4.1 \%$ of total body weight for young rats and $2.8 \%$ of total body weight for old rats. The liver weight was verified by direct weighing at the end of the perfusion. Experimental flow rates were found to be $1.0 \pm 0.1 \mathrm{ml} /$ min per $\mathrm{g}$ wet liver weight.
Perfusate volumes were adjusted on an estimated liver weight basis to maintain a volume proportional to liver size in both young and old rats (volume $=4.0 \mathrm{ml}$ times the weight of the perfused liver). This volume adjustment was made in order to compare the changes in perfusate insulin concentration and $t_{1 / 2}$ that were observed in livers from young and old rats (see Calculations).

All livers were perfused initially for $30 \mathrm{~min}$ to remove endogenous insulin, after which a single dose of porcine insulin (Iletin, U-100, E. Lilly Co.) was added to the perfusate reservoir to achieve an initial of $1000 \mu \mathrm{U} / \mathrm{ml}$. Perfusate aliquots of $0.8 \mathrm{ml}$ were withdrawn from the perfusate reservoir at $0,5,10,15,30,45$ and $60 \mathrm{~min}$. Insulin removal was measured over the physiological range between 600 and $15 \mu \mathrm{U} / \mathrm{ml}$, in which the insulin concentration showed a linear regression in relation to time.

\section{Calculation of Insulin Removal}

Hepatic insulin clearance $(\mathrm{k})$ was calculated from the following formula [6]:

$$
\mathrm{k}=\frac{\operatorname{Vol} .(\mathrm{ml}) \times \ln \frac{\mathrm{C}_{\mathrm{a}}}{\mathrm{C}_{\mathrm{f}}}}{\mathrm{dt}(\mathrm{min})}
$$

where $C_{a}$ and $C_{f}$ are initial and final insulin concentrations over the time interval dt. This equation allows stepwise adjustment for volume change resulting from sampling loss. A $\mathrm{k}$ value is computed for each successive time interval during which the insulin concentration continues to fall at a linear rate. This interval was from 10 to $45 \mathrm{~min}$ in young rats and from 10 to $60 \mathrm{~min}$ in old rats. A mean hepatic clearance $\bar{k}$ is obtained for each perfusion by averaging the individual values for $\mathrm{k}$

As derived by Mortimore et al. [6], the mean hepatic clearance $\overline{\mathrm{k}}$ provides a quantitative approximation of the kinetics of insulin removal by the perfused liver. This constant is a direct function of liver mass and is calculated from the two factors, Vol and $\frac{\ln \mathrm{C}_{\mathrm{a}} / \mathrm{C}_{\mathrm{f}}}{\Delta \mathrm{t}}[6,8]$. When control and experimental livers of similar size are perfused with comparable perfusate volumes, the volume factor becomes constant for both, and the $\mathrm{k}$ is directly proportional to $\frac{\ln C_{\mathrm{a}} / C_{\mathrm{f}}}{\Delta \mathrm{t}}$, or its derived expression $\mathrm{t}_{1 / 2}$ [7]. To compare insulin removal by livers of different size, mean hepatic clearance $\bar{k}$ should be expressed per unit liver weight.

$$
\text { 1) } \overline{\mathrm{k}} / \mathrm{g} \text { liver }=\mathrm{Vol} \times \frac{\ln \mathrm{C}_{\mathrm{a}} / \mathrm{C}_{\mathrm{f}}}{\Delta \mathrm{t}} / \mathrm{g} \text { liver }=\text { Vol } / \mathrm{g} \text { liver } \times \frac{\ln \mathrm{C}_{\mathrm{a}} / \mathrm{C}_{\mathrm{f}}}{\Delta \mathrm{t}} \text {. }
$$

By maintaining the $\mathrm{Vol} / \mathrm{g}$ liver ratio relatively constant at $4 \mathrm{ml} / \mathrm{g}$ liver weight, differences in insulin removal $(\overline{\mathrm{k}})$ by livers from old and young rats are also directly proportional to $\frac{\ln C_{a} / C_{f}}{\Delta t}$, or the half-time of insulin removal from the perfusate. Insulin half-time $\left(t_{1 / 2}\right)$ was calculated from the formula of Burgi et al. [7].

2) $\mathfrak{t}_{1 / 2}(\min )=\frac{\ln 2 \times \operatorname{Vol}(\mathrm{ml} / \text { gliver })}{\bar{k}(\mathrm{ml} / \mathrm{min} / \text { gliver })}$

with mean perfusate volume and $\mathrm{k}$ expressed per unit liver weight.

\section{Analytical Methods}

All blood and perfusate samples were centrifuged immediately after collection, and the serum was stored at $-20^{\circ} \mathrm{C}$. Glucose was analyzed by electronic measurement of the rate of $\mathrm{O}_{2}$ consumption during glucose oxidase reaction (Glucose Analyzer, Beckman 
Table 1. Mean ( \pm SEM) values for body and liver weights, fasting plasma glucose and insulin concentrations, and insulin $t_{1 / 2}$

\begin{tabular}{|c|c|c|c|c|c|c|c|c|}
\hline Study & Group & $\begin{array}{l}\text { Number } \\
\text { of } \\
\text { rats }\end{array}$ & $\begin{array}{l}\text { Weight on day } \\
\text { of perfusion } \\
\text { (g) }\end{array}$ & $\begin{array}{l}\text { Liver wt. } \\
\text { (g) }\end{array}$ & $\begin{array}{l}\text { Daily } \\
\text { wt. gain } \\
\text { (g) }\end{array}$ & $\begin{array}{l}\text { Fasting } \\
\text { glucose } \\
(\mathrm{mg} / 100 \mathrm{ml})\end{array}$ & $\begin{array}{l}\text { Fasting } \\
\text { insulin } \\
(\mu \mathrm{U} / \mathrm{ml})\end{array}$ & $\begin{array}{l}\text { Insulin }\left(t_{1 / 2}\right) \\
(\min )\end{array}$ \\
\hline \multirow[t]{2}{*}{ A } & Young (control) & 7 & $178 \pm 5$ & $7.3 \pm 0.6$ & $7.4 \pm 0.4$ & $90 \pm 4$ & $18 \pm 4$ & $7.62 \pm 0.29$ \\
\hline & Old (control) & 10 & $601 \pm 10^{b}$ & $16.9 \pm 0.5^{b}$ & $0.7 \pm 0.4^{b}$ & $104 \pm 5$ & $33 \pm 5^{a}$ & $11.00 \pm 0.56^{b}$ \\
\hline \multirow[t]{2}{*}{ B } & Young (control) & 7 & $175 \pm 6$ & $7.3 \pm 0.6$ & $7.4 \pm 0.4$ & $93 \pm 3$ & $17 \pm 3$ & $8.12 \pm 0.64$ \\
\hline & Young (Ins) ${ }^{c}$ & 7 & $180 \pm 4$ & $7.5 \pm 0.3$ & $6.8 \pm 0.3$ & $45 \pm 4^{b}$ & $87 \pm 15^{b}$ & $8.59 \pm 0.41$ \\
\hline \multirow[t]{2}{*}{$\mathrm{C}$} & Old (control) & 7 & $598 \pm 8$ & $16.8 \pm 1.0$ & $0.7 \pm 0.3$ & $99 \pm 4$ & $31 \pm 5$ & $11.44 \pm 0.54$ \\
\hline & Old $\left(\mathrm{SZ}_{22}\right)^{\mathrm{c}}$ & 7 & $593 \pm 11$ & $16.8 \pm 0.5$ & $0.3 \pm 0.3$ & $191 \pm 9^{b}$ & $17 \pm 2^{\mathrm{a}}$ & $11.12 \pm 0.57$ \\
\hline \multirow[t]{4}{*}{$\mathrm{D}$} & Young (control) & 15 & $175 \pm 4$ & $7.6 \pm 0.5$ & $7.5 \pm 0.5$ & $90 \pm 3$ & $18 \pm 3$ & $7.71 \pm 0.31$ \\
\hline & Young $\left(\mathrm{SZ}_{50}\right)^{\mathrm{c}}$ & 7 & $181 \pm 8$ & $7.0 \pm 0.2$ & $4.4 \pm 0.4^{\mathrm{a}}$ & $171 \pm 7^{a}$ & $10 \pm 1^{a}$ & $8.18 \pm 0.45$ \\
\hline & Young $\left(\mathrm{SZ}_{80}\right)^{\mathrm{c}}$ & 11 & $162 \pm 5$ & $6.2 \pm 0.3$ & $0.4 \pm 0.2^{b}$ & $400 \pm 12^{b}$ & $<2.0^{\mathrm{b}}$ & $5.55 \pm 0.35^{b}$ \\
\hline & Young $(\mathrm{CR})^{\mathrm{C}}$ & 6 & $171 \pm 2$ & $6.7 \pm 0.2$ & $0.2 \pm 0.3^{b}$ & $94 \pm 4$ & $15 \pm 3$ & $5.86 \pm 0.51^{b}$ \\
\hline
\end{tabular}

a Denotes significant difference from control $(\mathrm{p}<0.05)$

${ }^{b}$ Denotes significant difference from control $(\mathrm{p}<0.01)$

c Description of animal treatments:

Ins $=$ Administration of NPH insulin for 12-14 days

$\mathrm{SZ}_{22}=$ Administration of streptozotocin, $22 \mathrm{mg} / \mathrm{kg}$ body weight

$\mathrm{SZ}_{50}=$ Administration of streptozotocin, $50 \mathrm{mg} / \mathrm{kg}$ body weight

$\mathrm{SZ}_{80}=$ Administration of streptozotocin, $80 \mathrm{mg} / \mathrm{kg}$ body weight

$\mathrm{CR}=$ Caloric restriction to prevent weight gain

Instruments, Fullerton, Calif.). Insulin concentrations were determined by modification of the immunoassay procedure of Desbuquois and Aurbach [9].

\section{Results}

The data in Table 1 (A) indicate that old rats weighed more, had bigger livers, and gained less weight per day than young rats. In addition, they had higher fasting insulin levels than did young rats. The fall in perfusate insulin concentrations over time when livers from these rats were perfused is illustrated in Figure 1, and the linear regression of the insulin concentration between 600 and $15 \mu \mathrm{U} / \mathrm{ml}$ is apparent in this figure. Within this interval of linear descent, the mean hepatic clearance $\bar{k}$, and the insulin half-life, $t_{1 / 2}$, were calculated from formulas 1 and 2 , for each perfusion experiment.

The $t_{1 / 2}$ of insulin removal by perfused livers from the old and young rats of Study $A$ are listed in Table 1 and illustrated in Figure 2. These results demonstrate that the $t_{1 / 2}$ of the older rats was significantly prolonged (11.0 vs $7.6 \mathrm{~min}$ ).

In an effort to see if the prolongation of the $t_{1 / 2}$ of insulin removal by perfused livers of the older rats was due to the coexisting increase in fasting insulin level, hyperinsulinaemia was produced in young rats by the chronic (12-14 days) administration of insulin. This resulted in a fall in fasting glucose level and an approximate 5-fold increase in fasting insulin level as seen in Table 1 (B). However, in spite of this elevation of insulin level, the $t_{1 / 2}$ of insulin removal by

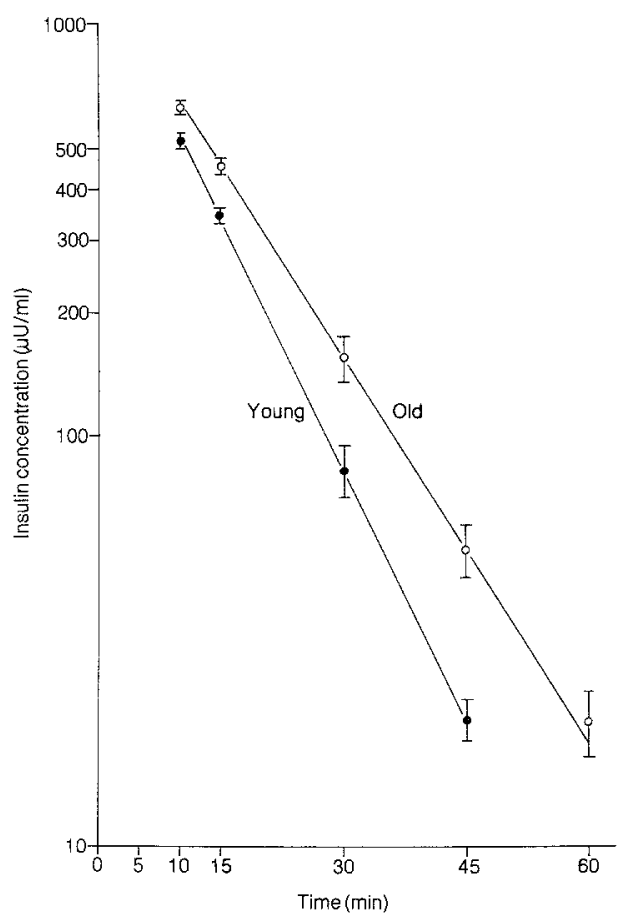

Fig. 1. Changes in insulin concentration during liver perfusions in young and old rats. Each point represents the mean ( \pm SEM) of at least seven experiments, and the plot demonstrates a linear regression of the insulin concentration with time. The mean hepatic clearance $(\overline{\mathrm{k}})$ is derived from the above data by determining the $\mathrm{k}$ values that obtain between each sampling time. For example, by using the data above and equation 1 , the successive $\mathrm{k}$ values of young rats were $2.14(10-15 \mathrm{~min}), 2.50(15-30 \mathrm{~min})$, and 2.39 $(15-45 \mathrm{~min})$, resulting in a $\overline{\mathrm{k}}$ of $2.37 \mathrm{ml} / \mathrm{min} / \mathrm{g}$ liver. Substituting this value in equation 2 , and the mean perfusate volume of $26.0 \mathrm{ml}$, results in a calculated mean $\mathrm{t}_{1 / 2}$ of $7.6 \mathrm{~min}$ for insulin removal by perfused livers of young rats 


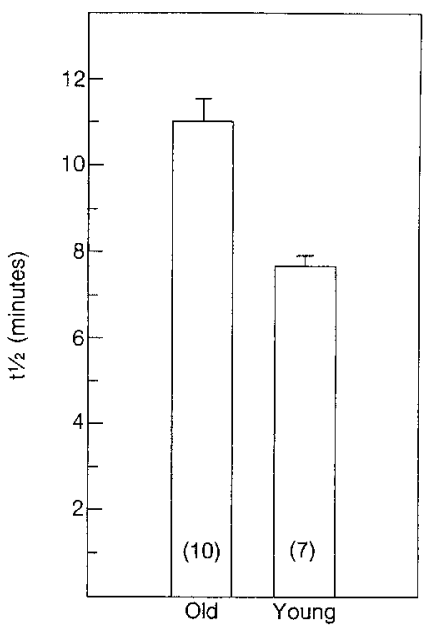

Fig. 2. Mean ( \pm SEM) half-time $\left(t_{1 / 2}\right)$ of removal of insulin by perfused livers from old rats as compared to livers from young rats

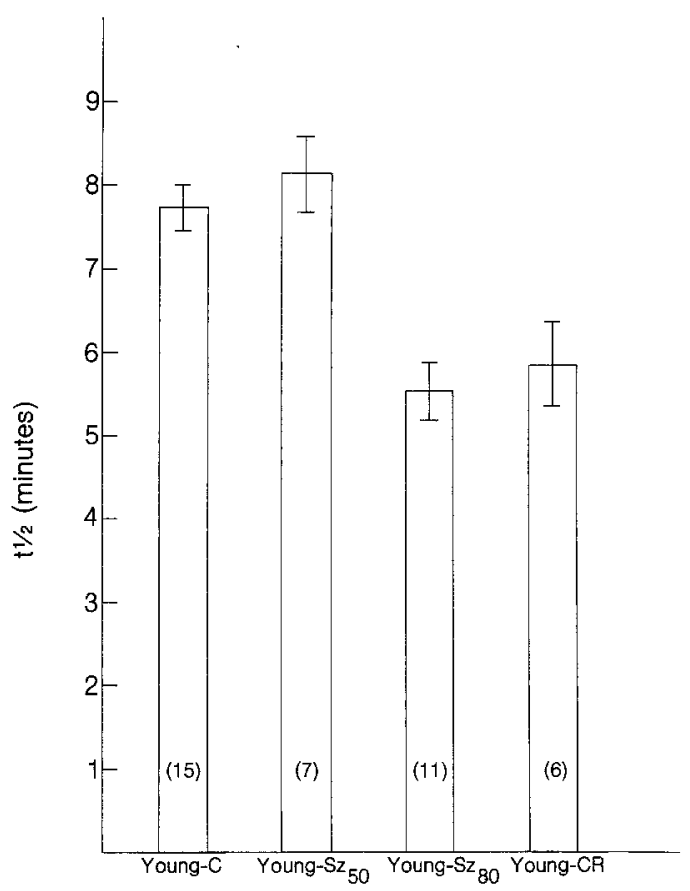

Fig. 3. Mean ( \pm SEM) half-time $\left(t_{1 / 2}\right)$ of insulin removal by perfused livers from young rats injected with either 50 (Young-SZ or 80 (Young-SZ 80 ) $\mathrm{mg} / \mathrm{kg}$ of streptozotocin, or livers from calorically restricted (Young-CR) as compared to livers from young control rats (Young-C)

livers from young, hyperinsulinaemic rats was unchanged from that of young, NPH diluent fluid injected controls [Table 1 (B)].

In order to further evaluate the effect of ambient insulin level on the $t_{1 / 2}$ of insulin removal, streptozotocin $(22 \mathrm{mg} / \mathrm{kg}$ body weight) was injected into old rats $\left(\right.$ Old-SZ $\left.\mathrm{SZ}_{22}\right)$. This produced an approximate doubling of fasting glucose concentration, but the weight gain of the SZ-treated old rats was comparable to that of the same aged control animals [Table 1 (C)]. The data in Table 1 (C) also demonstrate that Old-SZ $\mathrm{SZ}_{22}$ rats were significantly hypoinsulinaemic as compared to old control rats. However, this decrease in fasting insulin level did not lead to a change in $t_{1 / 2}$ of insulin removal by these streptozotocin-treated old rats, and it remained prolonged [Table 1 (C)].

In order to further explore the proposed relationship between circulating insulin level and insulin removal rate, insulin deficiency of varying degrees was produced in young rats by injecting different doses of streptozotocin. The hyperglycaemia and hypoinsulinaemia produced by $50 \mathrm{mg}$ of streptozotocin per $\mathrm{kg}$ body wt in these young rats is seen in Table 1 (D). This dose of streptozotocin again resulted in an approximate doubling of fasting glucose concentration, and the rate of weight gain in these rats was slightly, but significantly less than that of the controls. Although significant hypoinsulinaemia developed in these rats, there was no change in the $t_{1 / 2}$ of insulin removal [Table 1 (D) and Figure 3]. However, when young rats were injected with $80 \mathrm{mg}$ of streptozotocin per kg body weight, severe hyperglycaemia and hypoinsulinaemia were produced, and this was associated with an absolute absence of weight gain [Table 1 (D)]. In this instance, Table 1 (D) and Figure 3 , there was a significant fall in the $t_{1 / 2}$ of insulin removal. However, Table 1 (D) and Figure 3 also indicates that a similar fall in $t_{1 / 2}$ was observed in the young rats subjected to caloric restriction. The insulin levels of the young calorically restricted rats were equal to normal controls, and significantly lower $(\mathrm{p}<0.01)$ than those of rats with severe diabetes. On the other hand, rats treated with $80 \mathrm{mg}$ of streptozotocin per $\mathrm{kg}$ body weight and those subjected to caloric restriction were equally "catabolic", as judged by a similar failure to gain weight [Table 1 (D)]. These results suggest that it was the catabolic state, not hypoinsulinaemia per se, that seemed to account for the shortening of the $t_{1 / 2}$ of insulin removal in the severely diabetic (and hypoinsulinaemic) rats.

\section{Discussion}

The results presented indicate that perfused livers from one year old Sprague-Dawley rats remove insulin less efficiently than do perfused livers from young control rats. A similar decrease in rat of hepatic insulin removal was noted by Karakash and colleagues when they compared ob/ob mice to a control group of nonobese mice [1]. In both instances the defect in hepatic insulin removal was detected in experimental 
animals who were both hyperinsulinaemic and obese as compared to the control population. Our observations indicate that the defect in efficiency of hepatic insulin removal is not confined to a unique genetic variant, but may be a general feature associated with obesity and/or hyperinsulinaemia.

The cause of the observed defect in hepatic insulin removal, as well as the relative role played by hyperinsulinaemia as compared to obesity in the genesis of the abnormality, remains to be clarified, Karakash and colleagues found that the administration of streptozotocin, anti-insulin serum, or simple starvation all led to partial correction of the impaired insulin removal in perfused livers of ob/ob mice. Since all of these procedures also led to a fall in circulating insulin level, the suggestion was made that the defect was secondary to the hyperinsulinaemia. Support for this hypothesis can also be found in the earlier work of McCarroll and Buchanan [10] who noted that isolated perfused livers of rats whose ambient insulin levels were lowered by streptozotocin or a three day fast had significantly increased hepatic insulin removal when compared to normal fed controls. However, our results suggest that the situation may not be that simple. Thus, we were not able to modify the rate at which insulin is removed by simply varying insulin level over relatively short periods of time (1-2 weeks). Neither increased insulin levels in young animals, nor decreased insulin levels in old and young animals led to any change in hepatic insulin removal, as long as some degree of positive caloric balance was maintained. The only situation in which insulin deficiency led to an increase in hepatic insulin removal was that associated with severe diabetes in young rats who were unable to gain weight. Since the rate of hepatic insulin removal increased to the same degree when young rats were calorically restricted, in the face of normal insulin levels, it seems likely that it was the catabolic state, and not the insulin deficiency, which led to the change in $t_{1 / 2}$.

In conclusion, these data demonstrate that the rate of hepatic insulin removal declines as rats get old and obese. These findings are very similar to those previously reported for the ob/ob mouse [1], and suggest that this defect in hepatic insulin removal may be a general feature of the obese state. Although the cause of this abnormality is unclear, it does not seem to be a simple function of ambient insulin level. Whatever the cause, it is clear that this defect could make a significant contribution to the hyperinsulinaemia of the human obese state.

Acknowledgements. This work was supported in part by a grant from the National Institutes of Health, \#HL 08506, and from the Research Services of the Veterans Administration.

\section{References}

1. Karakash, C., Assimacopoulos-Jennet, F., Jeanrenaud, B.: An anomaly of insulin removal in perfused livers of obese hyperglycemic (ob/ob) mice. J. Clin. Invest. 57, 1117-1124 (1976)

2. Zucker, T. F., Zucker, L. M.: Fat accretion and growth in the rat. J. Nutr. 80, 6-19 (1963)

3. Bracho-Romero, E., Reaven, G. M.: Effect of age and weight on plasma glucose and insulin responses in the rat. J. Am. Geriatr. Soc. 25, 299-302 (1977)

4. Mondon, C. E., Olefsky, J. M., Dolkas, C. B., Reaven, G. M.: Removal of insulin by perfused rat liver: effect of concentration. Metabolism 24, 153-159 (1975)

5. Mondon, C. E., Burton, S. D.: Factors modifying carbohydrate metabolism and effect of insulin in perfused rat liver. Am. J. Physiol. 220, 724-734 (1971)

6. Mortimore, G. E., Tietze, F., Stetten, D.: Metabolism of insulin- $\mathbf{I}^{131}$ : studies in isolated, perfused rat liver and hindlimb preparations. Diabetes 8, 307-314 (1959)

7. Burgi, H., Kopetz, K., Schwartz, K., Froesch, E. R.: Fate of rat insulin in rat liver perfusion: studies by adipose tissue assay. Lancet 1963 II, 314-316

8. Brauer, R. W.: Liver circulation and function. Physiol. Rev. 43, 115-213 (1963)

9. Desbuquois, B., Aurbach, G. D.: Use of polyethylene glycol to separate free and antibody bound peptide hormones in radioimmunoassays. J. Clin. Endocrinol. Metab. 33, 732-738 (1971)

10. McCarroll, A. M., Buchanan, K. D.: Insulin clearance by the isolated perfused livers of insulin deficient rats. Diabetologia 9, 457-460 (1973)

Received: April 24, 1978,

and in revised form: August 15, 1978

Gerald M. Reaven, M. D.

Veterans Administration Hospital (111-M)

3801 Miranda Avenue

Palo Alto, CA 94304

USA 